\title{
Culture and Birthing: Experiences from a Rural Community in Western Kenya
}

\author{
Abraham Rono ${ }^{1, *}$, Harisson Maithya ${ }^{2}$, Benard Sorre ${ }^{1}$ \\ ${ }^{1}$ Department of Anthropology and Human Ecology, School of Arts and Social Sciences, Moi University, Kenya \\ ${ }^{2}$ Department of Sociology and Anthropology, School of Humanities and Social Sciences, South Eastern Kenya University, Kenya
}

Copyright $\odot 2018$ by authors, all rights reserved. Authors agree that this article remains permanently open access under the terms of the Creative Commons Attribution License 4.0 International License

\begin{abstract}
Despite numerous campaigns to increase uptake of hospital delivery services in Kenya, skilled birth attendance is still low. The situation is particularly worse for marginalized rural communities, including the Marakwet of western Kenya. Marakwet County ranks far below the national health survey indicators, with only $28 \%$ of the women delivering in hospitals compared to the average national index, which is at $62 \%$ [1]. Different reasons are cited for the low health facility use by women during delivery. Drawing from a study on determinants of birthing sites conducted among the Marakwet of western Kenya, this paper discusses the socio-cultural factors that influence women's choice of the site of birthing. The study employed both qualitative and quantitative methods of data collection and analysis and it reports the qualitative findings. A total of 186 mothers, selected using multistage cluster sampling, were interviewed. Three focus group discussions with participants ranging from 8-12 were conducted, one with mothers below 20 years of age, another with mothers above 45 years of age and the third one with married men. A total of 29 participants participated in the FGDs. The FGDs participants were separately selected and were outside the 186 sampled informants. The study found that traditional birth attendants, rituals performed in the birthing process, and disposal of the placenta were the critical socio-cultural factors influencing the Marakwet women choice of birthing site. The paper concludes that understanding and addressing these factors is the key to any intervention programs designed to improve the reproductive health of women through the utilization of hospital as a birthing site.
\end{abstract}

Keywords Birthing Site, Socio-cultural, Decision Making, Marakwet, Kenya

\section{Introduction}

According to World Health Organization, more than $90 \%$ Watemi ethnic groups, the husbands make a choice of of all maternal mortalities occur in the less developed countries where deliveries are often conducted by unskilled birth attendants. Globally, existing body of literature points to various social and cultural factors that have been attributed to low access to health facilities by expectant women. In a study done in Delhi, lack of social support for childcare emerged as one of the primary reasons as to why women choose home as place of delivery [2]. In Indonesia, one factor that influenced the use of traditional birth attendants (TBAs) was advice by other family members such as the older sisters, parents, or husbands on where to deliver. Also, a long-time tradition in the community of using the services of traditional birth attendants, who had been the only service providers during birthing for many years before the National Health System started, was also considered a reason why community members use the services of TBAs during childbirth. There was a perception that delivery is a natural rite of passage for women and, therefore, home delivery was preferred unless complications occurred or someone persuaded them to deliver at health facilities. It was also reported that being part of the community, speaking the local language, living in the community, and sharing the same culture meant that TBAs commanded trust in the community [3]. Anthropologist Arthur Kleinman's explanatory models (EMs) propose that individuals or groups of individuals may have vastly different notions of health and disease. What a physician sees as an illness or condition that requires medical attention may not be necessarily so for the sufferer and his or her close kins whose theories of disease causation are different. In effect, health care providers have neglected a very crucial aspect, the patient's perspective or the culture within which the individual lives. This partly is the reason why some studies describe antenatal care and hospital delivery as unnecessary medicalization of pregnancy and birthing [4].

In Ghana, community groups including churches were also cited as advising women to seek a health care facility delivery [5]. In Tanzania, among the Maasai and the
Watemi ethnic groups, the husbands make a choice of 
where the wives should deliver. The Maasai women reported that they shy away from hospital delivery because they perceive vaginal examinations as painful and could damage the baby. The Watemi women say that it is dehumanizing for a male healthcare provider to perform a vaginal examination and this makes most of the women to choose home deliveries where all traditional birth attendants are women. In addition, the Watemi community holds a strong belief that the woman should resume normal responsibilities of caring for the other children and household chores as soon as possible after delivery [6]. Thus, women's own knowledge, their social responsibilities, and gender of the health care providers influence the choice of birthing site. Elsewhere a study done in Rakai, Uganda showed that significant others played a crucial role in determining the choice of a woman's birthing site. The study found that the husband and relatives made the choice of where a woman delivers [7].

Among the Akamba of southern Kenya, a combination of factors makes home delivery attractive. Many women opt for birthing at home because they consider it a familiar environment and comfortable. They also consider home delivery convenient and inexpensive. An equally important factor that keeps women away from hospital delivery is the seemingly indiscriminate use of caesarean section to deliver women. Women consider this as unnecessary medicalization of their reproductive life. Birthing among the Akamba is believed to be normal if a woman is allowed to labour since pain endurance is an important element of motherhood and it is a fulfilling experience [8].

A study that used key informant interviews conducted with TBAs and hospital staff in Malindi revealed that many Swahili and Giriama women consider it a taboo to prepare for birth anywhere because they believe it would result in misfortunes including still birth. The preparations mentioned include buying clothes, purchase of birthing supplies like razor blade, setting aside money for delivery and deciding on where to deliver. Also it is considered harmful to guess the sex of the baby or give a name or a gift to the unborn baby. These discourage women from making individual birthing plans, including delivery at a health facility [9]. Thus studies are not agreed on the factors that contribute to communities in general and women in particular to prefer one birthing site and not the other.

There is consensus by healthcare providers that maternal deaths can be prevented if all deliveries are overseen by skilled birth attendants in a health care facility [10], but this may not be realized in most parts of the rural settings in Kenya, including the study area, where most women deliver at home under medically inadequate conditions. The underlying reasons why most rural communities in Kenya including the Marakwets deliver at home have not been fully investigated and documented. Previous studies have focused on maternal mortality per se but have not addressed the reasons why women do not choose health care facilities as birthing sites. This paper is an output of a study that was carried out by the authors on determinants of birthing site among women in a rural community in Western Kenya. Whereas health professionals consider health care facilities as ideal for deliveries, the socio-cultural factors that influence the choice of birthing site is a knowledge gap, which the current paper seeks to address.

\section{Materials and Methods}

\subsection{Study Design}

A descriptive cross sectional design was used. This design provides information about naturally occurring behaviours, attitudes or other characteristics of a particular group at a given period in time. The study on which this paper draws was designed to obtain in part the sociocultural factors that influence the choice of women's birthing site among the Marakwet community of Kenya.

\subsection{Study Area}

The study was conducted in Marakwet in western Kenya. The specific research area was Endo Ward in Kerio valley in Marakwet East Sub County. Marakwet East Sub County and Marakwet West Sub County were split from the former Marakwet District. Marakwet East Sub County is bordered by West Pokot County to the North, Baringo to the East, Trans-Nzoia to the West and Marakwet West Sub County to the South. It covers an area of $1588 \mathrm{~km}$ square with a projected population of 191,988 persons. Over half of the population in Marakwet is poor, with $52.7 \%$ living in absolute poverty [11]. A combination of poverty, difficult terrain, harsh climate, poor infrastructure, and scanty public resources has left the area lagging behind in terms of socio-economic development. Majority of the Endo residents are subsistence farmers. They keep livestock such as cattle, goats, sheep and chicken. Endo residents also grow crops, among them, mangoes, paw paws, bananas, cassava, and green grams. The study area has six healthcare facilities: one County hospital with no operating theatre, two government health centres and three dispensaries. The healthcare facilities are evenly distributed in the area but a low utilization of hospital delivery services has been a persistent challenge in the area.

\subsection{Research Type}

The research combined both qualitative and quantitative methods of data collection and analysis. Qualitative method of data collection was used to collect data on socio-cultural factors influencing the choice of birthing site while quantitative method was used to collect and rank those factors in order. 


\subsection{Research Duration}

The study was conducted over a twelve-month period starting from $14^{\text {th }}$ August 2013 and ending on $13^{\text {th }}$ August 2014. Additional visits for data update were done in September 2016.

\subsection{Target Population}

The target population of this study comprised women who had delivered prior to the execution of the study. Compared to women who had never delivered, the target study population had the experience of having delivered at a chosen birthing site.

\subsection{Inclusion Exclusion Criteria}

\subsubsection{Inclusion Criteria}

$\begin{aligned} \text { i. } & \text { Mothers who had delivered at least once } \\ \text { ii. } & \text { Mothers above } 18 \text { years of age } \\ \text { iii. } & \text { Should have been living in Endo Ward, Marakwet } \\ & \text { East Sub County }\end{aligned}$

\subsubsection{Exclusion Criteria}

i. Mothers who had never delivered

ii. Mothers below 18 years of age

iii. Mothers who live outside Endo Ward, Marakwet East Sub County

\subsection{Sampling}

A sample size of 186 was calculated using Fischer's Formula below:

$$
\mathrm{n}=\frac{\mathrm{Z}_{\alpha}^{2} \mathrm{PQ}}{\mathrm{d}^{2}}
$$

Where:

$\mathbf{n}$ is the sample size

$\mathrm{Z} \alpha$ is the normal variate associated with significance level $\alpha$ (1.96 is the normal deviate associated with $95 \%$ confidence interval)

$\mathbf{P}$ is proportion of estimated population if the target population if less than 10,000 . The estimated population of women of childbearing age in Endo Ward was 5,927, hence the assumption that the number of women who had delivered before was less than 10,000. Also, the proportion of women who delivered in hospitals was 14 percent from DHIS 2012/2013 Financial Year, hence $\mathbf{P}=0.14$.

$$
\mathrm{Q}=1-\mathrm{P}
$$

d is the required level of precision / discrepancy Therefore,

$$
\begin{aligned}
& \mathrm{n}=\frac{(1.96)^{2} \times 0.14 \times 0.86}{(0.05)^{2}} \\
& =185.01(\mathbf{1 8 6} \text { women })
\end{aligned}
$$

Multistage cluster sampling was used to select the specific respondents who participated in the study. The researchers identified the five locations in Endo Ward and used them as clusters. The specific respondents were then selected using simple random sampling procedure, which was proportionate to size of the population from each of the clusters. Numbers representing every household in the Community Health Strategy Household register (for each location) were written in pieces of paper, folded and mixed in a closed carton. The researchers then picked numbers representing households from each location and the female in that household who met the set criteria was selected as the respondent. The same procedure was followed until the sample size of 186 was reached. This method allowed for equal and non-zero chance of inclusion and representation of the respondents in the target study population.

Purposive sampling procedure was used to select four key informants and the Focus Group Discussion participants for the study. Two In-charges of health facilities and two traditional birth attendants were selected to participate as key informants. Three Focus Group Discussion (FGD) were held; one with women who delivered before reaching 20years, another one with older women who delivered after attaining 45 years of age, and the last one was carried out with married men whose wives had given birth at least once. Each FGD had between 8 and 12 participants. Thus a total of 29 participated in the three FGDs. This method enabled the researchers to capture the views and experiences of the young women, older generation of women who had given birth more than once, and the married men.

\subsection{Data Collection Methods and Materials}

\subsubsection{The Structured Interview}

Data were collected using an interview schedule for 186 respondents who were randomly sampled. The questions generated data on the socio-demographic factors, socio-cultural factors and healthcare systems factors, which influenced the choices women make regarding birthing site. The survey method was also suitable for quantitative and some qualitative data for the study objectives.

\subsubsection{Key Informant Interviews}

A key informant guide was used. Key informant interviews with two traditional birth attendants (TBAs), and two In-charges of health facilities were conducted using interview guides to collect in-depth qualitative data. This method gathered in-depth information from the TBAs and health care providers in health facilities. It took the form of face-to-face interview between the researchers and the key informants and this allowed for clarification and detailed explanations of their attitudes and perceptions about birthing site.

\subsubsection{Focus Group Discussions.}

Focus group discussions were conducted with two 
groups of women: one with 9 women who delivered after 45 years of age and another one with 12 women who delivered when they were teenagers (before 20years). Women who delivered when they were teenagers but had exceeded 20 years at the time of FGDs were excluded. Focus Group Discussion was suitable because it allowed the researchers to gain an in-depth understanding of variations or similarities in the Marakwet women's choice of birthing sites at the two extremes of the reproductive ages. The third FGD was conducted with 9 married men whose wives had delivered before. Thus a total of three focus group discussions were conducted. This allowed the researchers to understand the role of men in the choices that women make or choices that are made for them regarding birthing site.

\subsection{Methods of Data Analysis}

The study utilized both quantitative and qualitative methods of data analysis. Descriptive statistics were generated from quantitative data using SPSS Version 22. Frequencies, percentages, and measures of central tendencies are employed to present the data in the form of tables, graphs and charts. These statistics are used to summarize the characteristics of the respondents including age, gender occupation, marital status, and household size. Cross tabulations are also used to check for any association between birthing site, which is the dependent variable and the independent variables. Qualitative data were analysed by deriving explanations and interpretation of the findings based on identified themes. The qualitative data are presented as narratives in the form of quotes, narrations and summary statements.

\section{Results}

This paper, therefore, presents findings on the socio-cultural factors influencing women's choice of birthing site among the Marakwet of Western Kenya. According to the Marakwet community, when a woman is four months pregnant, she is expected to visit a traditional birth attendant. The visits will then continue on a monthly basis. During these visits, the TBA examines the mother by palpating the abdomen to assess the 'position' and state of the baby in the uterus. The four-month threshold, before visiting the TBA, was chosen because the TBAs believe that it is at four months that they can confirm that a woman is pregnant, as captured by the following observation:

When a woman comes for examination before four months, I cannot be sure that the big abdomen is a baby. Some women come and when you palpate there is nothing. But after four months, you can feel with your hands that there is something, and you can sometimes feel it moving (63 years old TBA, Endo Ward).
Once a woman is confirmed to be pregnant, the Marakwets have taboos, which serve as norms that guide the behaviour of the woman until she gives birth. For instance, she is expected to stop having sex. Among this community it is also considered a taboo for a pregnant woman to view the body of a dead person. In this regard, a pregnant woman is prohibited from attending funerals. If she attends, then she is not allowed view the body of the dead person because this is likely to cause misfortunes.

The study also found that it is forbidden for pregnant women to eat meat from a dead animal including goat, cow, sheep, chicken and other domestic animals. The only meat they are allowed to eat is from a slaughtered animal. Traditional herbs are taken by pregnant women to ensure that they are, together with their babies, healthy. These herbs are composed of traditional roots, which are boiled and the women drink on a daily basis. The pregnant women are also given special milk, mursik, which is sour milk mixed with blood. This is believed to boost their immunity and body strength.

A special necklace (see figure 1) is also worn by a pregnant woman with charms to guard the pregnancy from miscarriage and evil eyes, Thus the Marakwet believe that these charms confer protection to both the mother and the unborn baby. However, when the woman experiences labour pains, the necklace is removed to allow the woman to give birth. The community believes that if the special necklace is not removed, the woman will not give birth due to obstruction or she will have protracted labour. The foregoing finding was near universal with $93 \%$ of the respondents reporting that the Marakwet have traditional objects used by TBAs to assist in the birthing process. Figure 1 shows the special necklace with a pouch containing charms that is worn by pregnant women.

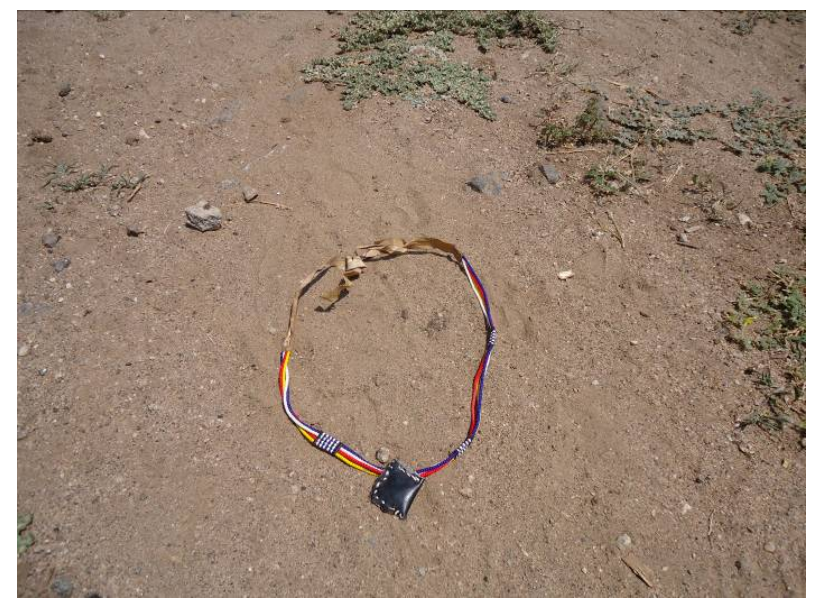

Figure 1. The special necklace worn by a pregnant woman

Regarding the birthing site, the study found that a pregnant woman delivers in a small house called kobo kogo or in a kitchen. During this process, a therapy management group is constituted and is composed of mothers who have delivered before and the traditional birth attendant. Those 
women who have not given birth, but have been circumcised, are also allowed to be part of the therapy management group. The delivery is performed by the traditional birth attendant and the other members present only play a supportive role. The women who have given birth before and those who have been circumcised offer psychological support and encourage the woman in labour to endure the labour pain and to push during delivery. The men are relegated to the periphery in the birthing process, and only provide resources and a safe environment for birthing.

When the baby is born, the TBA uses a razor blade to cut the umbilical cord, and then waits for the placenta to expel, which she disposes while observing a culturally defined ritual. One FGD participant described it as follows:

The placenta is taken carefully by the TBA and laid in a specified manner; millet is spread along the way as she goes to dispose it. This will ensure that the woman does not become infertile. It is then laid in the bush, then milk is poured on it and left... It should not be buried (51 years old woman FGD participant).

In an interview with a key informant, similar explanation was provided thus:

The placenta is taken to the bush, then held by the cut umbilical cord and laid as millet is spread on the ground...for a male child it is taken to the right hand direction from the house of delivery and for a girl it is taken to the left hand direction. (59 years old TBA, Endo Ward).

When the process of delivery is complete, an abdominal belt, leketio is tied to the abdomen to aid involution of the uterus and to guard the child from harm. The leketio is made of animal skin and cowrie shells as shown in figure 2:

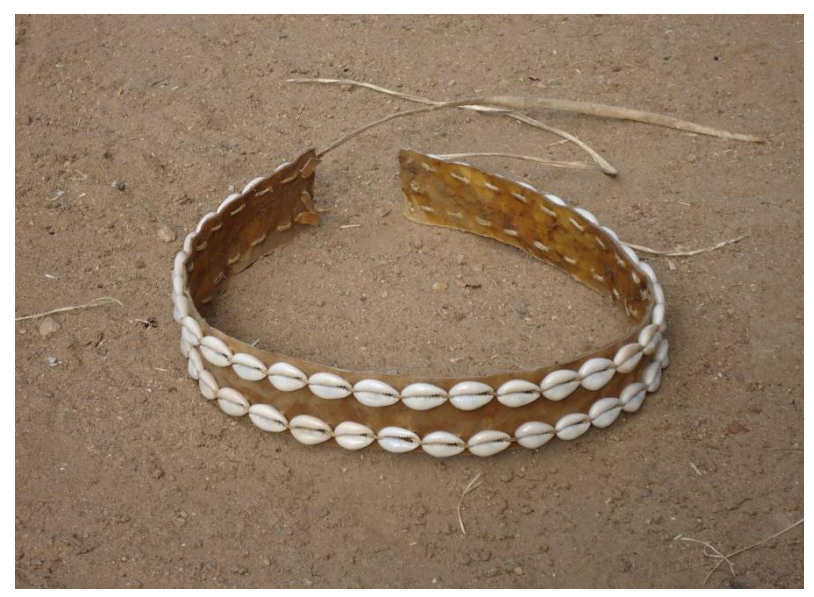

Figure 2. A leketio

The woman is then secluded in the house for six months postpartum and she is fed on special diet, which includes meat and herbs to replace the blood lost during delivery. Where possible, a goat is slaughtered for her, because the special diet is thought to make recovery faster. After seclusion, the newly delivered woman goes through a cleansing ritual before interacting with other members of the community. The ritual involves taking a bath using boiled herbs. Traditional liquor is brewed and a drinking and celebration party held. It is after this is completed that the post-natal woman is allowed to interact with other community members and the baby is allowed to be seen and held by other community members.

Female circumcision is also a factor that plays a role in determining where a woman chooses to deliver. For women who have undergone female circumcision, they choose to deliver at home under TBAs because often, the TBAs are the ones who performed the circumcision. On the contrary, if the women chose to deliver in hospital, they fear that they will face stigma and prejudice from nurses in the healthcare facilities. Treatment by the health care providers discourages them from choosing hospital as a birthing site.

The nature of pregnancy also influences the choice of birthing site. In the Marakwet community, there is a widely held belief that if a woman conceived out of wedlock, she is likely to experience a difficult labour. If that happens it is expected that the husband or age mates of the husband would perform a ritual to 'free' the baby to be born. A key informant observed,

If a woman gets pregnant out of wedlock she uses her money to go to hospital, if the husband is angry with her, the baby will not come out until he spits saliva on her so that the baby can come out...but if that is not done, operation [Caesarean Section] in hospital will have be performed on her (63 years old TBA).

When hospitals were introduced in the study area, there were no implicit or explicit efforts to encourage or discourage the use of hospitals as birthing site. It was assumed that hospital will be the natural site. But as one TBA observed, hospitals became just one of the available options of birthing sites that the woman may choose for delivery.

To triangulate the key informant findings with other sources, supplementary socio-cultural data were elicited from the general respondents who were asked to rate their responses on a five point Likert Scale. Responses for these factors ranged from 1 to 5 points where $1=$ strongly disagree while $5=$ strongly agree. The mean was used to assess the scores. The closer the mean is to 5 , the more it was agreed that the factor influences the choice of birthing site. Similarly, the closer the mean is to 1 , the more the extent of disagreeing with the statement.

Taboos associated with the disposal of placenta and presence of traditional objects used by TBAs to assist the birthing process, were reported to influence the choice of birthing site. The notion that women who deliver from hospitals are socially stigmatized was strongly disagreed upon by most of the respondents. The findings are summarized in table 1. 
Table 1. Sociocultural factors and their ranked influence on birthing site

\begin{tabular}{|c|c|c|c|}
\hline Socio-cultural factors & Mean & Std. Deviation & Rank \\
\hline Marakwet community has taboos associated with disposal of placenta & 4.94 & 1.104 & 1 \\
\hline The Marakwet community have traditional objects used by TBAs to assist birthing process & 4.77 & 1.108 & 2 \\
\hline The birth order (whether first or subsequent) influences the choice of birthing site & 4.37 & 1.378 & 3 \\
\hline Marakwet community has food habits which regulate pregnancy and birthing & 4.32 & 1.393 & 4 \\
\hline The Marakwet community have preferred birthing sites ( where births ideally occur) & 4.31 & 1.419 & 5 \\
\hline The choice of birthing site is socially prescribed for the women in Marakwet community & 4.22 & 1.559 & 6 \\
\hline Men (Husbands) have no role in the choice of birthing site & 4.16 & 1.631 & 7 \\
\hline $\begin{array}{l}\text { The birthing site chosen defines whether one is a true Marakwet woman ( through } \\
\text { circumcision and other rites of initiation) }\end{array}$ & 1.92 & 1.729 & 8 \\
\hline $\begin{array}{l}\text { The nature of pregnancy (out of wedlock, within marriage, planned or unplanned) influences } \\
\text { the choice of birthing site. }\end{array}$ & 1.87 & 1.807 & 9 \\
\hline Women who deliver from hospitals are socially stigmatized & 1.54 & 1.909 & 10 \\
\hline $\begin{array}{c}\text { There are cultural rites and rituals performed for those who deliver in hospital or otherwise } \\
\text { places. }\end{array}$ & 1.26 & 1.937 & 11 \\
\hline
\end{tabular}

From table 1, taboos associated with the disposal of placenta, was considered the major factor influencing choice of birthing site with a mean of 4.94. Presence of traditional objects used by TBAs to assist in birthing process with a mean of 4.77 was ranked second in terms of the level of influence on the choice of birthing site. The factor with the least influence on the choice of birthing site was social stigmatisation of women delivering in hospital with a mean of 1.26 .

\section{Discussion}

In the Marakwet community, pregnancy, childbirth and postpartum practices are culturally prescribed and form a journey that every woman would go through. Marakwet women may shun hospital delivery because it is a drift away from this social and culturally acceptable journey. There are multiple norms that the women are expected to observe. One such norm is abstinence from sex when they are pregnant. The pregnant women are warned that if they have sex, the baby will be born covered with 'white dirt' that is believed to be the semen from the man. Such a woman faces social stigma because she is perceived as having no sexual control, which is the reason she had sex when she was pregnant. These findings are similar to those of a study conducted in Tanzania where infidelity is believed to result in punishment from God. Similarly, curses from elders may lead to death of the pregnant woman and those who attend to her during birth [6]. However, in other communities, sex during pregnancy is not entirely prohibited. Among the Akamba community of southern Kenya sex is permitted in the early stages of pregnancy because semen is believed to nourish the mother and the baby [8].

In the present study, it is a taboo for a pregnant woman to view the body of a dead person. Breaking this taboo may have fatal or undesirable consequences. It is believed that if she views a dead body, the baby will die in the uterus. A non-fatal but undesirable outcome is that the baby will be born with congenital abnormalities. While this taboo may not present immediate physical danger to the baby, it may have health implications. It discourages pregnant women from emotionally draining circumstances like grieving, which may trigger premature labour resulting in preterm delivery hence endangering the survival of the new baby. More broadly, these notions are informed by aetiologic concepts of illnesses and misfortunes in this community, indeed, many African communities.

Eating meat from a dead animal is prohibited. This is because it is believed that eating a dead animal may harm the mother and/or the baby in the uterus and leads to death. This socio-cultural practice is consistent with the scientific knowledge that meat from a dead animal could potentially spread infection. The researchers argue that this is a norm to guard against eating meat from infected animals that is dangerous to her health and that of her unborn baby. Beliefs regulating what a pregnant woman eats were also observed among Indian women who believe that eating excessive food can make the baby too big leading to difficult labour. Nutrition-related practices during pregnancy are based on a belief that 'hot' foods are harmful and 'cold' foods are beneficial. Because pregnancy is believed to generate a hot state, pregnant women are advised to maintain a balance by eating cold foods and avoiding hot foods. Cold foods are recommended in early pregnancy to prevent miscarriage. Hot foods are encouraged during the last stages of pregnancy to facilitate labour. Fiji-Indians believe that it is the responsibility of the others to satisfy the cravings of a pregnant woman because a baby who dribbles excessively indicates that the mother was not taken care of properly during pregnancy [12]. Similar food restrictions exist in the Akamba community of Kenya. Traditionally, pregnant women are discouraged from eating certain foods. Such foods include eggs, fatty meat, and honey so as to avoid the baby 
becoming too big because that would cause problems during delivery, which may result in the death of the mother, the baby or both. An overlarge baby would require delivery procedures that were traditionally unavailable. In the contemporary society, women are still advised to avoid such foods in the advanced stages of pregnancy [8]. Such restrictions serve to maintain or foster good health in an environment of limited health care resources.

A pregnant Marakwet woman is expected to take certain herbs. The community believes that these herbs make the woman strong so that she will have the energy to push the baby for a normal delivery at birth. The herbs are also believed to build and boost blood levels because blood loss is anticipated at birth. This is also thought to prevent anaemia. A similar practice of taking herbs was reported in a study done among women seeking healthcare at Dilokong hospital in Limpopo, South Africa. In that context, herbs called joko tea are used consequent to a belief that it wards off evil spirits [12]. Further, some Indian women may take herbs to promote development of a male foetus [13]. Delivery requires body 'strength' and these findings are consistent with the findings of a study among the Akamba of southern Kenya who believe that strength is an important indicator of reproductive health [8]. On the whole a pregnant woman is considered vulnerable both physically and socially and these norms and practices are intended to offer protection to both the woman and the unborn baby.

The ideal birthing site in the community is the kobo kogo, $a$ small house. It is considered inconvenient to deliver in the main house because it is used by the husband, other family members and visitors. This small house is not only convenient but also it offers privacy comparable to that found in a maternity ward.

Women who have delivered before and the circumcised women are allowed to be present during delivery. This is because they are considered to be mature and accepted as adults. Those who have not delivered and those who have not been circumcised are viewed as children and, therefore, not allowed into the delivery room. More important is the fact that those women who have the birthing experience can provide support that may be needed during delivery.

A ritual of spreading millet and pouring of milk is conducted during disposal of the placenta. The direction followed is determined by the sex of the child. In the ritual, the spreading of millet symbolizes fertility and a propagation of generations. The milk poured as libation is meant to appease the ancestors so that the child is protected from any harm. The right hand is associated with strength and authority, while the left hand is associated with weakness and submission. That is why the placenta of a male child is taken to the right hand direction from the birthing site, and that of a female child is taken to the left hand direction of the birthing site. Thus the proper management and disposal of the placenta will contribute to the health and general wellbeing of the mother and baby, and by extension the community.

In many parts of the world, similar rituals exist on how the placenta is managed and disposed after birth. In Northern Sumatra, it is believed that the placenta hosts one of the seven souls the newborn child possesses, so great care and respect is taken when disposing it. Women in Hungary who desired to stop having children burnt the placenta and placed the ashes in their husband's' drink, while in Japanese culture it was believed eating the placenta would increase a woman's fertility. In Austria and parts of Italy, it was believed that the blood of a fresh placenta had medicinal value for everything from the removal of birthmarks to the cure for epilepsy. In some cultures, like some Native Hawaiian, Navajo and Maori tribes it is believed that by burying the placenta in the homeland, the child is bound to the land and his/her ancestral heritage. In Thai culture the placenta is salted, jarred and buried under a tree that corresponds to the symbol of the Asian year of the child's birth [14]. Thus the way placenta is disposed may impact on the wellbeing of the community.

During the postpartum period, the mother in the Marakwet community is fed on special diet of goat meat and sour milk (mursik) mixed with blood.to promote healing. The mother is also exempt from daily chores like cleaning, cooking and handling of food for the family. This is because body fluids (including blood and secretions from the birth canal after delivery) are believed to be dirty and can cause illness among other members of the family including the husband. Consequently, a newly delivered woman is secluded until she is dry and before she resumes her household duties. This is in consonance with postpartum practices among the Akamba of Kenya who give postpartum mothers special foods and beverages, which are taken hot. They include porridge made from finger millet or maize flour and beverages such as milo and tea. For the relatively wealthy and the local elite, bone meat soup, which is reach in iron and calcium, in addition to porridge, is given to the newly delivered mother. Soup from goat's meat is especially favoured as it is said to be rich in substances that restore the lost energy and blood because of the food that goats eat. Soup prepared from cleaned and boiled internal organs of a goat, such as intestines and the stomach is considered therapeutic [8].

From the foregoing discussion, the journey from conception to pregnancy and finally birth is culturally prescribed and institutionalized, forming part of the life experiences of a Marakwet woman. This pressure influences women to act in accordance with the Marakwet culture so that they are perceived as normal and acceptable in the society hence the preference for home deliveries.

\section{Conclusion s}

The aim of this paper was to examine and describe the 
socio-cultural factors that influence the choice of birthing site for the Marakwet women of western Kenya. It has described the sociocultural models that favour home as the birthing site. The paper concludes that the choice of birthing site among the Marakwet women is influenced by an interplay of multiple socio-cultural factors. These socio-cultural factors far outweigh other benefits that healthcare providers associate with hospital delivery. Any interventional community programs designed to improve the reproductive health of women particularly the utilization of hospital delivery services among the Marakwet community must take into account these factors. This body of knowledge needs to be applied when designing and implementing maternal and newborn care programs among rural communities in Kenya.

\section{Acknowledgements}

We wish to acknowledge our informants who provided invaluable data without which this paper would not have been possible. We acknowledge the support accorded to us by our colleagues who in one way or the other provided the opportunity to develop this manuscript. To you all we say thank you.

\section{REFERENCES}

[1] Kenya National Bureau of Statistics (KNBS) and ICF Macro (2010), Kenya demographic and health survey 2008-09. (2010), Calverton, Maryland: KNBS and ICF Macro.

[2] Niveditha, D. et al. (2014). Why women choose to give birth at home: a situational analysis from urban slums of Delhi, 2014. BMJ Open 2014; 4:e04401 doi:10.1136/bmjopen-2013-004401.

[3] Titaley, C., Hunter, C., Dibley, M., \& Heywood, P. Why do some women still prefer traditional birth attendants and home delivery? a qualitative study on delivery care services in West Java Province, Indonesia. BMC, 2010.

[4] Kleinman, Arthur. Patients and Healers in the Context of Culture. Berkeley, CA: Univ of California Press, 1980.
[5] Halley et al. Shifting norms: pregnant women's perspectives on skilled birth attendance and facility-based delivery in rural Ghana, African Journal of Reproductive Health March 2013; 17(1): 15, 2013

[6] Magoma et al. High ANC coverage and low skilled attendance in a rural Tanzanian district: a case for implementing a birth plan intervention, BMC Pregnancy and Childbirth, 10:13, 2010.

http://www.biomedcentral.com/1471-2393/10/13 (accessed on 11th April, 2013)

[7] Amooti-Kaguna B., \&Nuwaha F. Factors influencing choice of delivery sites in Rakai district of Uganda. Soc Sci Med 50(2):203-13, 2000

[8] Maithya, H. Sexual and Reproductive Issues among the Akamba of Kenya: Implication for the Management of Childbearing and STD and HIV/AIDS. Saarbrucken, Deutschland: VDM Verlag Dr. Muller Aktiengesellschaft \& Co. KG. Germany, 2009.

[9] Carter, A. Factors That Contribute to the Low Uptake of Skilled Care During Delivery in Malindi, Kenya, Independent Study Project (ISP) Collection. Paper 821, 2010 .

http://digitalcollections.sit.edu/isp collection/821(accessed on 20th December, 2012)

[10] World Health Organization. Partnership for Maternal, Neonatal and Child health Fact Sheets, 2011.

[11] Kenya Integrated Household Budget Survey, KEN-KNBS-KIHBS-2015-2016-v01, 2016

[12] Mogawane, M.A., Mothiba, T.M. \& Malema, R.N. 'Indigenous practices of pregnant women at Dilokong hospital in Limpopo province, South Africa', Curationis 38(2), Art, 2015.

http://dx.doi.org/10.4102/curationis.v38i2.1553 (accessed on $10 / 11 / 2017$ )

[13] Ushvendra Kaur Choudhry, Traditional practices of women from India: pregnancy, childbirth, and newborn care. Journal of Obstetric, Gynecologic and Neonatal Nursing, 26(5), 533-539, 1997.

[14] Burns Emily, Birthing Rituals. The Journal of Perinatal Education, 23(1),41-49 (2014)

[15] .http://www.pregnancy.com.au/birth-choices/birth-informati on/birth-articles/birthing-rituals.shtml (accessed on $23 / 11 / 2017$ ) 